\title{
Route planning for soft modes of transport: healthy routes
}

\author{
P. Ribeiro \& J. F. G. Mendes \\ Department of Civil Engineering, University of Minho, Portugal
}

\begin{abstract}
The application of sustainability principles on the definition of policies and actions for urban mobility relies on the promotion of less pollutant, economic, and more equitable modes of transport, such as walking and cycling, also known as smooth modes of transport. In this paper, a methodology is presented and described under the concept of healthy route generation. This integrates the contamination of the smooth modes of transport network, according to the noise levels and air pollution indices of the urban environment in which it is located, with the main objective of reducing the exposure level and the risk of development of respiratory and cardiovascular diseases. Healthy routes can be applied to attract and promote the use of smooth modes of transport in a regular basis in urban environments.
\end{abstract}

Keywords: urban mobility, sustainability, walking, cycling, route planning, healthy routes, air pollution, noise.

\section{Introduction}

Traditionally urban mobility policies are focused on the promotion of motorized traffic for all kind of trips. However, the process to achieve a more sustainable means of transport has shown that a new vision on urban mobility is being developed with the introduction of restrictions on motorized traffic while less pollutant and more efficient modes of transport must be used for travel, especially on a daily basis, such as walking and cycling. Usually in transportation planning, these are frequently named as "green modes", "nonmotorized modes", "healthy modes" and "soft modes" due to its intrinsic characteristics. Examples of good practices and accumulated experience in the promotion of the use of soft modes of transport can be found in some North 
European countries. However, it is generally accepted that there is a need for a deeper and broader research on the current cycling and walking urban contexts.

In global terms, a research area that has not been conveniently studied is related with the route planning specifically suited to soft modes of transport. Usually, route planners and GPS devices are more oriented for car users, with the main objective of the minimization of different variables, such as distance (shortest route), time (fastest route), or the global trip cost (less expensive route). When the focus users are pedestrians and cyclists, routing and navigation should incorporate other variables than those used for car users, such as safety, commodity and others mainly related with environmental issues, due to its intrinsic vulnerability in comparison with the motorized modes of transport.

This research aims at introducing in the trip decision process the influence of the principal environmental impacts which soft modes of transport are exposed to during their trips, mainly in urban environments, due to air pollution and noise levels. Commuters (e.g. children walking to school) or occasional (e.g. leisure walking) soft users could be irreversibly affected during their life because they travel through polluted urban axes of traffic due to the lack of information and awareness on the impacts that those everyday routes have in their cardiovascular and respiratory system.

In our information society, aspects related with environmental and health issues of the citizens of our urban environments have become determinant in the decision-making process of renting or buying a house, of finding a place to rest and live and, most importantly, of finding the best travel route with less damage to personal health. Consequently, in the near future, it is expected to be noted an increase in the demand for tools and devices related with route planning for soft modes of transport, which would allow the generation of alternative routes, specifically for these modes.

In this work, a methodology will be presented for the development of a route planner dedicated to soft modes of transport, considering the main environmental characteristics of the urban spaces in which they travel. Through the production of long-term maps of air pollution and noise, it is possible to obtain the necessary data for the development of an environmental impedance function to be applied in the circulation axes of the transport system, by means of the contamination of the distances of each generated route.

The minimization of the objective function related with the concentration and levels of certain pollutants would lead to the less polluted, less noisy or, in other words, more sustainable route, thus providing an environmentally and healthy better solution for pedestrians and cyclists. In sum, the main output of this work consists on a conceptual formulation of a model of generation of healthy routes for soft modes of transport to be used in digital planning devices, such as dedicated web platforms, and GPS navigation systems, resulting in an innovative and user-friendly solution for pedestrians and cyclists. 


\section{The role of soft modes of transport in urban transport systems}

\subsection{The use of soft modes of transport}

In most industrialized countries, people live mainly in urban environments. In Europe, this value represents around $60 \%$ of the population. On the other hand, a growing trend of the use of motor vehicles by commuters has been observed, especially in home-work and home-school trips, and those are mainly short trips of lengths below $3 \mathrm{~km}$. According to Figure 1a, the most efficient modes of transport for short trips, in terms of time spent per kilometer, are the soft modes of transport, especially cycling.

According to the World Health Organization, short trips taken in motorized vehicles should be potentially replaced by walking and cycling, with clear benefits for human health due to the achievement of permanent physical activity. For a long time, several studies related with public health risk have shown some inertia on the recognition of the global impact in human health of the generalized use of motorized traffic (e.g. accidents, air pollution and noise) for most activities in urban environments. However, this lack of use of soft modes of transport is already a new trend on practices and policies towards the achievement of a more sustainable urban mobility.

Walking and cycling have different rates of use across European countries, as shown in Figure 1b, and in this context Portugal occupies almost the end of the tail, especially when compared with countries such as Denmark or The Netherlands (EC [1, 2]).

a)

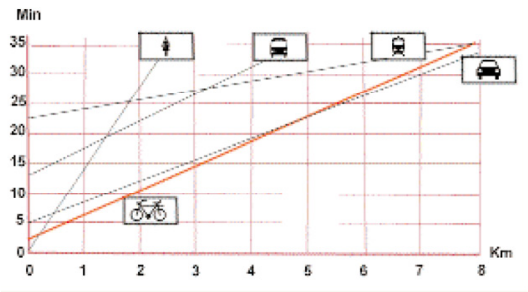

b)

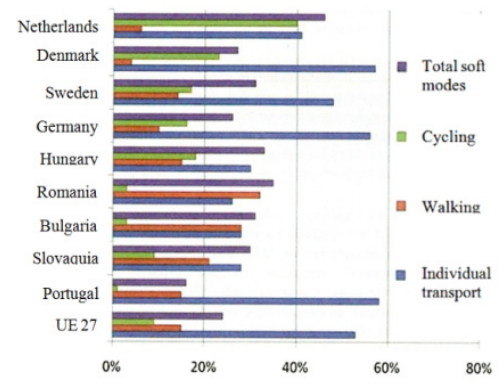

Figure 1: a) Travel time/length per mode of transport [1]; b) modes of transport used in the EU-27 [2].

Recently, several sustainable mobility plans for urban territories have been settled on different Portuguese municipalities. It was observed that a new paradigm was implemented for the establishment of local mobility policies through the adoption of actions oriented to the promotion of soft modes of transport over motorized traffic. In some case studies, it was perceived that there is a strong potential to attract more users for walking and cycling in most daily journeys (APA [3]). 
The proportion of adult population suffering from obesity, overweight or inactivity has grown in accordance with car dependency. Complementarily, there is an agreement and acceptance about the impact of motorized traffic on Earth's global warmth, as well as on environmental issues related with air pollution and traffic noise at a local level. Thus, it is necessary to encourage a change of the current patterns of urban mobility through the replacement of the more pollutant and less sustainable modes, such as cars, by alternative environmental friendly modes of transport, which contributes for a healthy society as well as the promotion of a greater social equity.

\subsection{Soft modes of transport and sustainability}

It is generally accepted that one of the most important issues of the cities of industrialized countries is the lack of sustainability of their transport system. Sustainability is an important vector that guides the implementation of functional and structural changes on consolidated urban spaces. Issues associated with climate changes, pollution and the reduction of natural resources are subjected to constant attention and concern by the public and have a growing impact on people's behavior, habits and consumption patterns. In Figure 2, the application of the sustainability concept on transport system is presented.

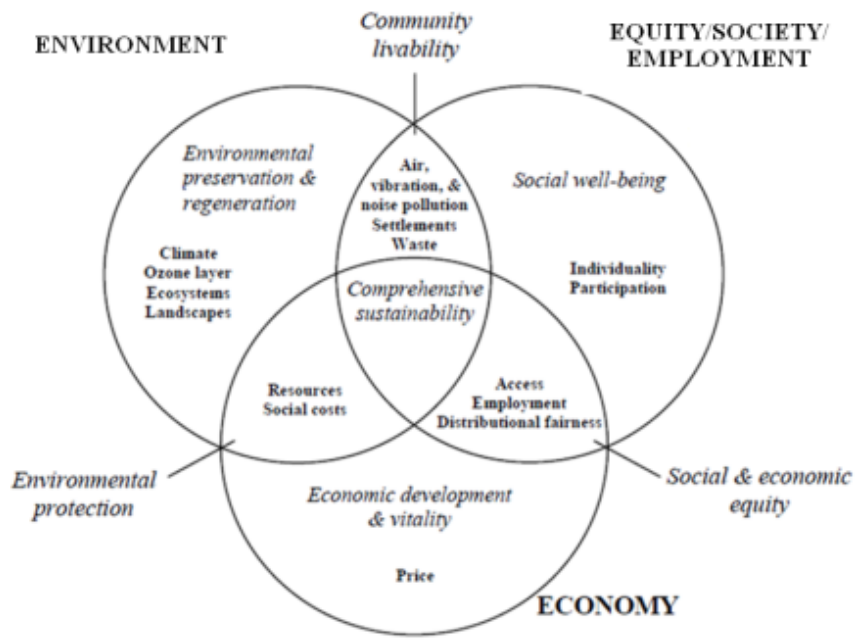

Figure 2: $\quad$ Sustainability applied to a transport system [4].

Thus, the promotion of living standards and more sustainable ways of travelling can be achieved through the introduction of significant improvements in the planning and design stages, taking into account the important contributions of several authors like Hall and Sussman [4], Jones et al. [5] and WCDE [6]. Moreover, it should be noted that studies on walking patterns revealed correlations between this mode of transport and the layout of cities as well as the age of dwellings. 
According to FHWA [7], the introduction of the bicycle was an important innovation for the promotion of social inclusion. Some studies show that when per capita income increases, the use of private car increases, while walking and cycling decrease. This situation is similar in some small and medium-sized cities of Portugal. For example, in the main parish of the village of Arcos de Valdevez, located in Northern Portugal, it was found that the proportion of trips made on foot decreased from $70 \%$ to $30 \%$ between 1991 and 2001 , and there was an increase in travel in private cars from $35 \%$ to $50 \%$ (Ribeiro et al. [8]).

Moreover, FHWA [7] identified a wide range of benefits ranging from environmental issues to improvements in the functioning of the transport system, related to the use of walking and cycling modes. The implementation of shortterm travels on foot or by bicycle can bring socio-economic, environmental and health benefits, which are evidenced mainly in the reduction of air pollutant emissions and in the congestion of urban road networks.

Several studies have shown significant benefits that derive from the regular and daily practice of physical exercise. Walking is the primary option to increase physical activity in sedentary populations (Morris and Hardman [9]). Even moderate or low levels of physical activity performed regularly can reduce the risk of coronary heart disease, diabetes, stroke and other chronic diseases.

Additionally, it reduces the costs related to health care, contributing to greater autonomy and independence of people, especially at advanced ages. On the other hand, walking allows a greater interaction between people, as they socialize during these trips, especially in comparison to private car travel.

In recent years, the European Union (EU) has supported projects that aimed at promoting the use of soft modes of transport through project funding within the 4th and 5th Framework for Research Program. The European project ADONIS (Analysis and Development of New Insights into the Substitution of Short Car Trips by Cycling and Walking) was one of the first international projects that compiled a series of measures to promote the use of these modes in Europe. Another European project deserving special attention is the WALCYING (How to Enhance WALking and Cycling instead of shorter car trips and to make these modes safer) which, like ADONIS, aimed at the promotion and safety of soft modes instead of cars for short trips. Furthermore, it is possible to find a wide set of information on other projects related with soft modes that were promoted by different European countries and the EU itself (e.g. PROMISIS, PROMPT, VELO.INFO, among others) in Transport Research Knowledge Centre.

It should be noted that part of the 7th European Program, which runs between 2007 and 2013, is oriented to promote a more sustainable transport system, in particular in the area of surface transports, which includes walking and cycling, through the establishment of a "greener" transport system, modal shifting and fluidity in main transport corridors. Thus, it is intended to ensure a more sustainable urban mobility and to increase the competitiveness of the sector.

Certainly, some measures and programs aiming at the promotion of the use of soft modes will focus on the improvement of infrastructure and facilities (EC [10]), mainly on footways and cycle lanes. However, this may be insufficient and lead to high cost solutions, without recurrence (increase of users). It is necessary 
that the promotion of the use of soft modes is efficient and effective, leading to an increase of frequent users, aware of their role in the achievement of an urban sustainable mobility.

Pedestrians and cyclists can represent a "green", environmentally friendly and healthy brand. For this, it is necessary to develop information and decisionmaking tools regarding the selection of routes, especially in urban areas.

\section{Information systems, planning and navigation for soft modes}

In the current transportation system, the user assumes the role of both subject and object, since this element represents the ultimate target of policies and strategies of the various public and private organizations working in the system, while simultaneously making the whole system work. Associated with the introduction of new technologies in the operation of the transport system, the information system (IS) is a key tool for the proper functioning of the entire mobility system, especially when it has a multimodal character.

The route planning is an increasingly common reality, both for long (intercities travel) or short (suitable for urban areas). Traditional paper maps have begun to be replaced by digital formats that incorporate the same type of information and in some cases allow some level of interactivity between user and map, as well as the use of algorithms for navigation.

In the recent past, the information system had an individual and rather specialized character for different modes of transport, thus the information, in most cases, was related to the location and main infrastructure layout of each mode. It is commonly accepted that information systems for transport and mobility tend to take a leading role in the implementation and adoption of more sustainable policies, especially those related to processes of user's modal shift for less polluting modes of transport.

It is expected that, given the current social, economic and environmental situation in the world, the use of alternative modes of transport becomes the first modal choice, instead of an alternative to private cars. However, it is necessary that people govern their daily mobility patterns by principles of equity and sustainability, which can only be acquired through education, training and, especially, with high quality information.

A properly informed public is capable of choosing the best travel option, although the selection process is usually very individual, and aims at achieving the most convenient journey for its purposes. In the case of trips exclusively taken with a single mode of transport, the public will try to get as much information about this mode of transport as possible. Traditionally, this could be obtained in a basic information system, where the information refers to the stops and schedules associated to a specific mode of transport, such as buses and trains.

Among different types of information systems that exist in Portugal, it is possible to highlight important logistical interfaces represented by websites, namely those by transpor, transporlis e itinerarium.net, for the entire country, Lisbon and Porto, respectively. Despite the multimodal nature of those systems, 
it was found that only the itinerarium considers the possibility of walking between modes or destinations.

Current users of transport systems seek clear and objective information, where access should be easy and if possible immediate, i.e. tailored to the needs of each individual. These features can only be provided by powerful information systems, capable of simultaneously satisfying a diverse group of users, with different expectations and reasons to travel. Thus, it is possible to conclude that, for an Information System to be efficient and equitable, it must have dynamic and integrated technological support tools for users, with interactive planning and navigation features for the various transport systems.

One of the most advanced examples in the use of information systems is the digital system produced by Transport for London (TfL), which in an evolutionary way over the years, has been presenting a set of information related with mobility management on various modes of transport available in one of the biggest cities of the world. In terms of innovation, the site presents a much more flexible route planner (optimizer) than those usually available in other countries, such as Portugal. In addition to the traditional options regarding origins/ destinations and transport modes that are available to users, a set of additional options is provided for selecting the most suitable route. These options fall clearly in the basic principles of sustainability for an urban mobility system, wherein it is given the opportunity to make journeys involving the use of soft modes (Figure 3).
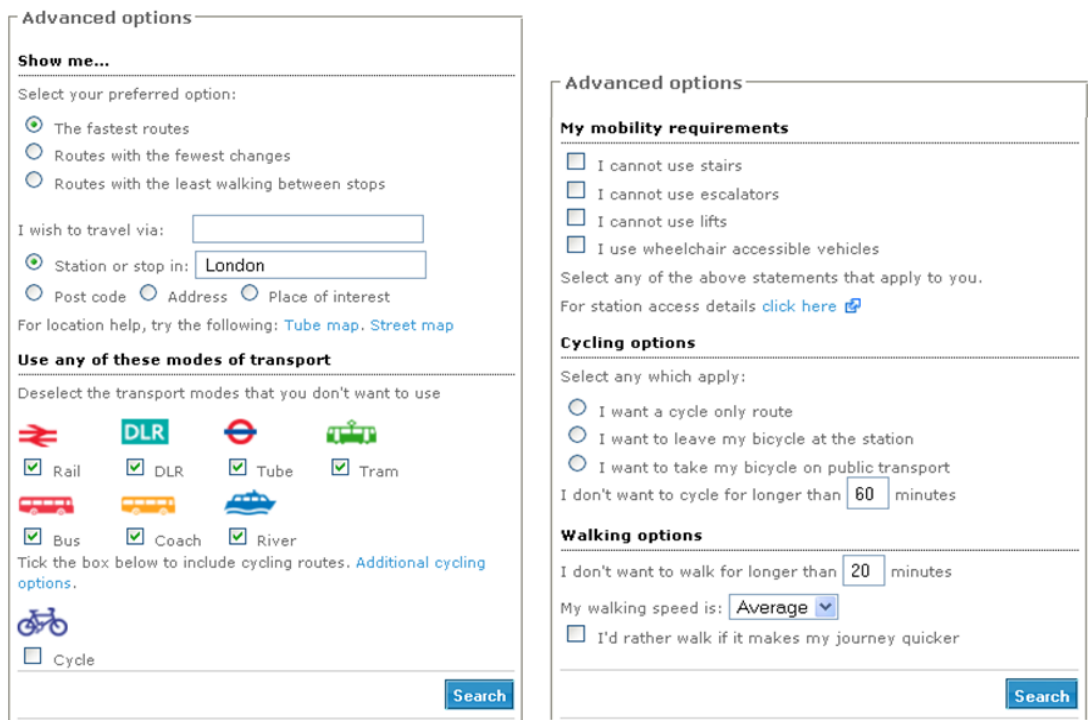

Figure 3: $\quad$ Example of the TfL route planner [11].

Most importantly, it enables the introduction of specific requirements that affect the mobility of pedestrians, especially for disabled people, as it is reflected in certain travelling options, namely wheelchair access, inability to use stairs or elevators. 
Given the current advances in terms of Information Systems, a new approach in route planning should include optimization criteria which should not be based only on issues related to the length and travel time, as is usually observed in systems planning and navigation for cars.

Therefore, this paper presents a methodology for the generation of routes integrating an innovative set of criteria appropriate to the reality of soft modes users, through the integration of environmental factors in the selection process, considering the impact of air pollution and noise, thus originating the so-called green/ sustainable and healthy routes.

\section{Healthy routes - methodology}

The definition of a route depends on several aspects related with the infrastructure and type of travelers. Thus, the concept of healthy routes, also sometimes referred to as green, clean, safe or sustainable routes for walking and cycling, considers in the definition of a itinerary a set of impedances of environmental nature and certain aspects associated with health, aiming to provide a comparison between different routes towards achieving the healthiest route.

The methodology to determine the healthy route is based on an information infrastructure, where basic data is collected and estimated through the simulation of noise levels and indices of air pollution. From this infrastructure, the relevant information will be extracted for each section of the transportation network (e.g. $69 \mathrm{dBA}, 10 \mathrm{mg} / \mathrm{m}^{3}$ for PM10, $70 \mathrm{mg} / \mathrm{m}^{3} \mathrm{NOx}$ ), which leads to the calculation of impedances, and consequently to a contaminated network which will be used in planning and navigation routes for soft modes of transport.

In general terms, the methodology has three distinct phases, which are: i) the generation of the pollution map; ii) the contamination of distances; iii) the generation of healthy routes, according to the diagram in Figure 4.

The generation of the pollution map associated with a particular cycling and walking network implies the estimation of noise levels and concentrations of various pollutants. These will be combined according to the level of impact that those pollutants have on human health, from which a single air pollution timedependent map will be produced, that will be extremely important for the second stage of the routing process.

The calculation of noise levels and concentration of pollutants requires the introduction of cartographic and road network data, as well as traffic. Therefore, it should be noted that the quality of the final output of the three phases of the routing process depends on the quality and control of the data collection.

Since the main scope of this work is the urban environment, the main source should be the motorized traffic, mainly due to the transfer of industries for specific areas around the outskirts of most cities. For this reason, the estimation of traffic volumes must be very carefully performed and, whenever possible, data should be obtained from daily traffic counts, even if allocation and distribution traffic models are going to be used during the estimation process of the pollutant emissions and noise. 


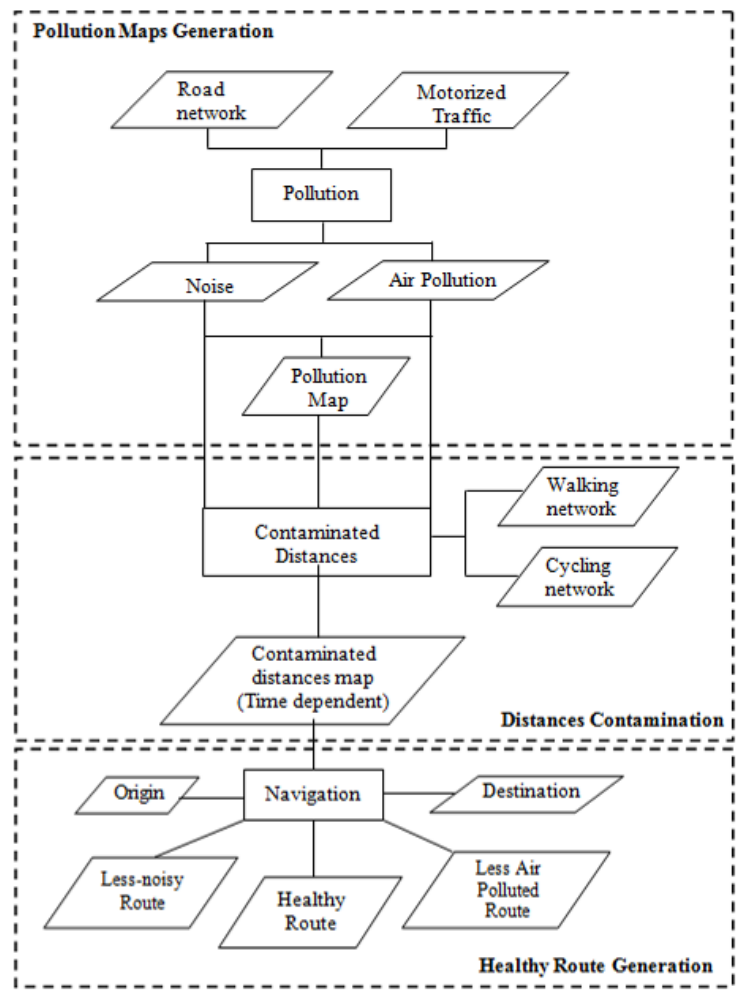

Figure 4: $\quad$ Flow diagram for the generation of healthy routes for soft modes.

Moreover, particular attention should be given to the type of software used to produce maps of noise and the various air pollutants under evaluation. The software should be capable of reproducing the typology of emissions produced in the urban road network under study, mainly to minimize the existing variability of the vehicle fleet in each country and, wherever possible, field measurements should be carried out to calibrate the models in which the pollution maps are based upon.

In the first phase, the production of maps of noise and air pollution is carried out with the use of specific software, in this case, CadnaA. This software is based on the AUSTAL program for the generation of emissions and a dispersion model of pollutants, which in turn originates from a particle dispersion Lagrangian model. On the other hand, the ESRI Arc Info software was used, since it allows operating with networks and traffic data, handling and treating the results of noise mapping and air pollution, in order to get the global pollution map for the selected areas (Figure 5a).

The second phase, related to the contamination of distances, refers to the creation of impedances to be assigned to all sections of the soft modes network, taking into account the data extracted from the pollution maps resulting from the first phase. However, it is important to refer that several pollution maps must be 
produced in order to obtain a global overview of the variability of the environmental conditions over space and time. For this, the production of hourly maps is recommended, or alternatively of maps in the most representative periods of the day (night, morning rush-hour, day, rush-hour afternoon and evening). In this stage of the routing process, it is essential to develop a GIS working platform to integrate the air pollution and noise information with the transport network facilities, and thereby build a base of contaminated network axes for each pollutant or for a combination of pollutants (Figure 5b).

a)
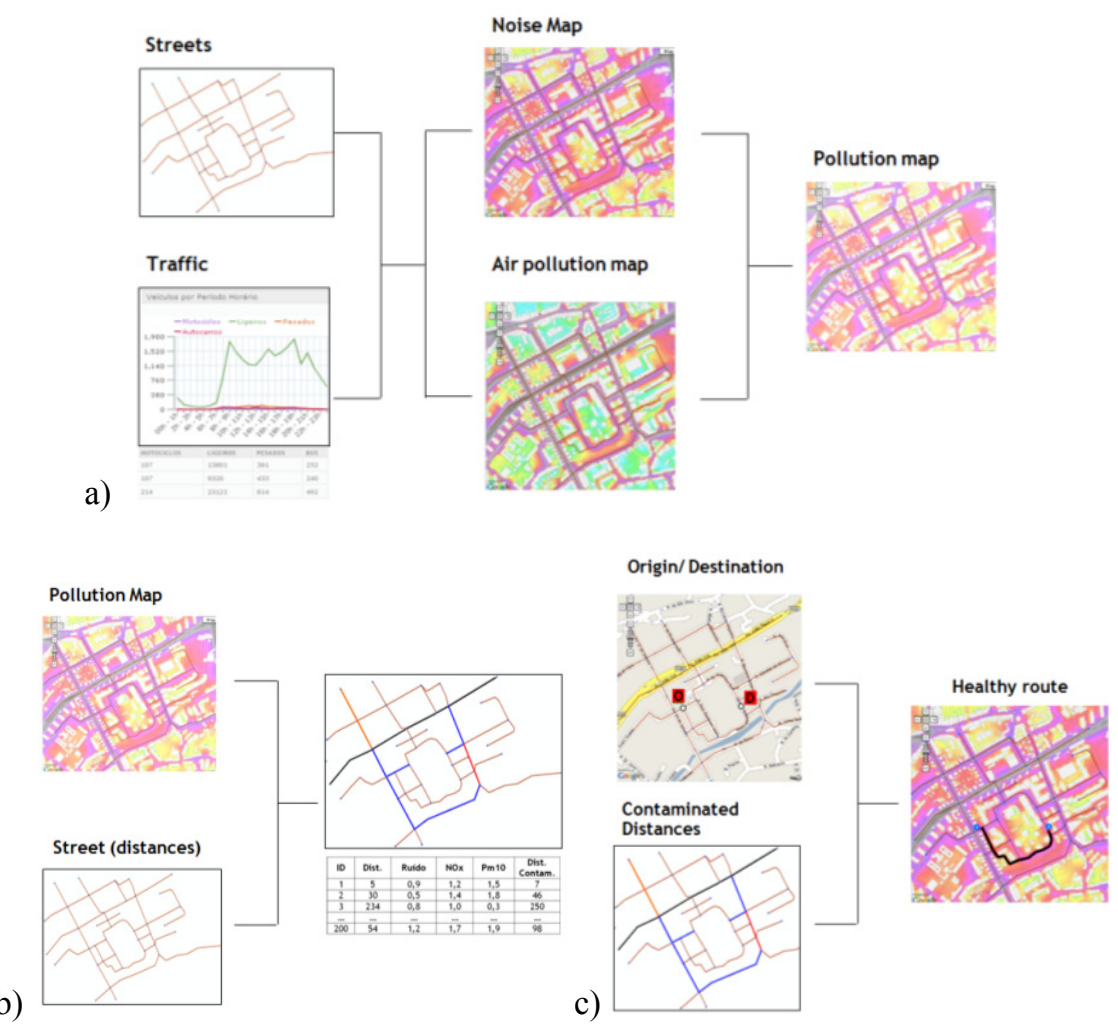

Figure 5: Schematic maps: a) generation of pollution maps; b) contamination of distances in terms of network axes; c) generation of healthy routes.

The last phase consists in making the generation process of routes and healthy itineraries operational, i.e. after having "contaminated" the soft modes network, a GIS database is produced with all contaminated distances (length of the axis). These are used in route optimization algorithms, in order to determine a wide range of routes, including the shortest, less noisy, with lower concentrations of air pollutants, and healthier route. To this end, there is a variety of commercial programs offering this possibility. In this work, the Network Analyst extension 
of ESRI's ArcInfo was used, which allows the production of several maps (Figure 5c) essential for planning and routing.

Traditional software tools have optimization processes aiming to determine the shortest route. In this work, the use of those algorithms serves to replace the actual distances of the branches of the network by properly contaminated axes. For this purpose, it is necessary to obtain relative values as a means to evaluate the impact of different pollutants in human health and its physical activity, which was done through the fusification of the pollutants. Moreover, it should be noted that there is a combined effect of different pollutants, even if its performance is studied separately. Thus, the validation and definition of thresholds and limit values for the various pollutants can be difficult, if not impossible to make. This complexity is even more significant when trying to account for how different individuals assimilate and react under various types of pollutants.

One of the main fields of application of this methodology of generation of healthy routes, herein presented, consists on the development of a decisionmaking tool and a route planning and navigation system exclusively for soft modes, namely through a website, in which it would be possible to get a set of spatial, functional and performance information for each displayed route. Thus, the pedestrians and cyclists can plan in advance the best route that suits its needs. Associated with the creation of the website, there is a strategy to achieve a stronger sustainable urban development, since it seeks to integrate options that allow the choice of the healthier and safer route for daily or sporadic activities, such as sports, among other applications. In a certain way, it intends to implement the idea that a good information system, easily accessible to the public, can contribute to reverse the current mobility patterns in the near future.

\section{Conclusions}

The current and future mobility policies should clearly incorporate the dimension of sustainability on the formulation of plans and on the implementation of actions to promote the use of soft modes in most urban trips.

For this purpose, this work proposed the generation of a route planner tool for soft modes of transport, taking into account the specific mobility needs of these modes in contrast to motorized transport. The entire process of conceptualization and achievement of the concept of healthy routes was presented using flowcharts and diagrams that contain examples of the street network maps and the associated pollution, which were preliminarily simulated for a city located in Northern Portugal.

The benefits of using technology resulting from the application of this methodology, which underlies the generation of healthy routes, can be observed in several situations. For example, for planning of home-school journeys for children who travel through urban areas, where high levels of noise, many cases above $75 \mathrm{dBA}$, as well as high levels of air pollution can be frequently observed. In the case of a child traveling in this kind of route every day by around $1 \mathrm{~km}$ (on average), at the end of the year the child would have travelled approximately 300 $\mathrm{km}$ under harmful health conditions, which may have a huge impact on the 
respiratory, cardiovascular systems, during this stage of life and even in adulthood. Thus, using a healthy route planner can minimize the effects of pollution on all citizens, with particular relevance to the most vulnerable groups of population, reducing potential health problems and associated costs.

\section{References}

[1] EC, Cycling: the way ahead for towns and cities. Brussels. http://ec.europa.eu/environment/cycling/cycling_en.pdf

[2] EC, Attitudes on issues related to EU Transport Policy. Brussels. http://ec.europa.eu/public_opinion/flash/fl_206b_en.pdf

[3] APA, Projecto de Mobilidade Sustentável. Manual de Boas Práticas para uma Mobilidade Sustentável. Agência Portuguesa do Ambiente. ISBN 978-972-8577-51-3, 2010.

[4] Hall R. P. \& Sussman J.M., Promoting the concept of sustainable transportation within the Federal System - The need to reinvent the U.S. DOT, TRB Paper 07-0565, 2006.

[5] Jones, P., Natalya, B. \& Marshall, S. Link \& Place-A Guide to Street Planning and Design. London. Landor Publishing. ISBN 1899650415, 2007.

[6] WCED, Our Common Future (Brundtland report), Oxford University Press, 1987.

[7] FHWA, Federal Highway Administration University Course on Bicycle and Pedestrian Transportation. US Department of Transportation, 2006.

[8] Ribeiro, P., Mendes, J.F.G. \& Fontes, A., A mobilidade sustentável em aglomerados de pequenas dimensões, Revista Minerva - Pesquisa \& Tecnologia. Volume 5 (2), pp. 149-158. ISBN: 978-85-85205-84-3, 2008

[9] Morris, J.N. \& Hardman, A.E., Walking to health. Sports Medicine. Vol. 23 (5), pp. 306-332, 1997.

[10] EC, Green Paper: Towards a new culture for urban mobility. http://ec.europa.eu/transport/clean/green_paper_urban_transport/doc/2007 0925 gp urban mobility en.pdf

[11] TFL, http://www.tfl.gov.uk 